\title{
Art or Science? An Evidence-Based Approach to Human Facial Beauty a Quantitative Analysis Towards an Informed Clinical Aesthetic Practice
}

\author{
Harpal Harrar $^{1} \cdot$ Simon Myers ${ }^{1} \cdot$ Ali M. Ghanem ${ }^{1}$
}

Received: 30 June 2017/ Accepted: 2 November 2017/Published online: 8 January 2018

(C) The Author(s) 2018. This article is an open access publication

\begin{abstract}
Background Patients often seek guidance from the aesthetic practitioners regarding treatments to enhance their 'beauty'. Is there a science behind the art of assessment and if so is it measurable? Through the centuries, this question has challenged scholars, artists and surgeons.

Aims and Objectives This study aims to undertake a review of the evidence behind quantitative facial measurements in assessing beauty to help the practitioner in everyday aesthetic practice.

Methods A Medline, Embase search for beauty, facial features and quantitative analysis was undertaken.

Selection Criteria Inclusion criteria were studies on adults, and exclusions included studies undertaken for dental, cleft lip, oncology, burns or reconstructive surgeries. The abstracts and papers were appraised, and further studies excluded that were considered inappropriate. The data were extracted using a standardised table. The final dataset was appraised in accordance with the PRISMA checklist and Holland and Rees' critique tools.

Results Of the 1253 studies screened, 1139 were excluded from abstracts and a further 70 excluded from full text articles. The remaining 44 were assessed qualitatively and quantitatively. It became evident that the datasets were not comparable. Nevertheless, common themes were obvious, and these were summarised.

Conclusion Despite measures of the beauty of individual components to the sum of all the parts, such as symmetry
\end{abstract}

Ali M. Ghanem

a.ghanem@qmul.ac.uk; academic-plastics@qmul.ac.uk

1 Academic Plastic Surgery Group, Blizard Institute, Barts and the London School of Medicine and Dentistry, Queen Mary University of London, 4 Newark Street, London E1 2AT, UK and the golden ratio, we are yet far from establishing what truly constitutes quantitative beauty. Perhaps beauty is truly in the 'eyes of the beholder' (and perhaps in the eyes of the subject too).

Level of Evidence $V$ This journal requires that authors assign a level of evidence to each article. For a full description of these Evidence-Based Medicine ratings, please refer to the Table of Contents or the online Instructions to Authors www.springer.com/00266.

Keywords Beauty · Facial measurements · Anthropometry · Facial beauty · Aesthetic - Quantitative analysis

\section{Introduction}

Facial aesthetic treatments have a significant influence on the individual and her perception of life. Differences in facial appearance provide individuality and are readily noticeable. The increasing appreciation of facial volume and tissue change has allowed the innovation and widespread use of fillers and the evolution of filling techniques, particularly fat grafting [1].

Demand for facial aesthetic treatments has increased in the last 20 years [2,3]. With this increase, it is becoming more important for the clinician delivering these treatments to understand what constitutes beauty and what motivates the patient to strive for beauty. Our self-perception of beauty has an impact on our everyday lives [4]. Others perceive a beautiful person to be more intelligent, sociable, friendlier and more desirable [5].

Many scholars throughout the centuries have debated what comprises beauty and indeed how to measure it in a standardised reproducible way [6]. Despite this centuries-old debate, there does not appear to be a validated, widely used 
set of evidence-based rules or measurements that can influence clinical practice. Understanding quantitative and objective features that constitute facial beauty is complex and confounded by multiple elements including society, culture, age and ethnicity [7]. Some argue that beauty is a myth and not reality and that the perception is learned and not developmental [8], and yet others argue that the perception of beauty is an innate developmental or biological ability [9]. Over the past few decades, the advancement of computer technology and computational capability may play a role in facilitating the assessment or evaluation of beauty. Differences in perceptions of facial aesthetics between professionals and patients have been well documented [10]. Preplanning, managing expectations and discussion of potential sequelae are already established protocols used by clinicians for a successful outcome for the patient. If standardised facial measurements could be incorporated into this process, it might allow the measurement of outcomes, have the potential to change the dynamics of a consultation and act as a useful consultation tool, to help manage expectations.

These measures based on evidence could be used as standards to guide the clinician. Based on the PICO framework [11], this study will aim to answer this research question-in the treatment of adults requesting facial aesthetic improvement, is there an evidence-based approach in quantitatively assessing beauty that is useful in everyday aesthetic practice?

\section{Methods}

A literature review was undertaken using Pubmed Medline, Medline Ovid, and EMBASE. Date limits were applied from 1970 to April 2017, and publications in English, humans and in peer-reviewed journals were included, with exclusions for abstracts presented at conferences. The search strategy was devised using three main concepts: (1) beauty AND (2) facial features AND (3) quantitative analysis (including terms proportions, distance, dimensions, length, height and width). Both thesaurus terms and text words (words or phrases appearing in the title or abstract of references) were identified for each concept. A manual check was undertaken given the sensitive nature of the search strategy used (use of quantitative analysis to aid in plastic or cosmetic surgical procedures to correct facial deformities or conditions); the search strategy is available.

\section{Inclusions}

Research papers, where adults were subjects, seeking facial aesthetic therapies or facial assessments were considered. Outcome variables of measured beauty parameters, facial
Table 1 Reasons for exclusion of full text articles

\begin{tabular}{ll}
\hline Reason for exclusions & Number \\
\hline $\begin{array}{l}\text { Dentofacial surgical correction/Le fort osteotomy } \\
\text { orthognathic }\end{array}$ & 14 \\
$\begin{array}{l}\text { Psychological effects of beauty/personality and beauty/ } \\
\text { brain effects on beauty }\end{array}$ & 12 \\
Inappropriate for other reasons & 8 \\
Orbital surgery/ear placement in reconstruction & 6 \\
Skeletal analysis & 5 \\
Cleft lip palate and surgery & 3 \\
Adolescent or child after manual records reviewed & 3 \\
Cancer surgery & 3 \\
Cosmetic or cosmeceuticals & 3 \\
Comparison of different fillers & 2 \\
Endoscopic lifting surgery & 2 \\
Burns victims/trauma victims & 2 \\
Qualitative measurements of facial aesthetic outcomes & 2 \\
DNA forensic analysis & 2 \\
Portrait painting theories & 2 \\
Cadaver & 1 \\
Total & 70 \\
\hline
\end{tabular}

measurements, ratios of measurements of the face, comparison of facial parameters were included.

Exclusions were applied for facial measurements undertaken for research on cadavers, burns and trauma victims seeking aesthetic treatments. Exclusions were also applied where plastic and reconstructive or dental surgery would have been the predominant procedure.

\section{Results}

A total of 182 entries had been considered as duplicates from the search of 1455 . The search criteria did not fully exclude articles with patients who were children or adolescents, and these were further excluded after reviewing the abstracts. Table 1 lists excluded articles and reasons for their exclusion. Of the remaining 44 studies, the full articles were extracted and checked. These were further scrutinised for their methodology and outcomes data. Due to the diversity of the types of studies, combining them was not appropriate statistically although some grouping was possible according to common themes (Fig. 1, Table 2).

\section{Discussion}

Measurements of facial proportions introduced by the Greeks, the Classical Canons and later adopted by the Renaissance artists, the Neoclassical Canons are used by 
Fig. 1 Study protocol flow diagram

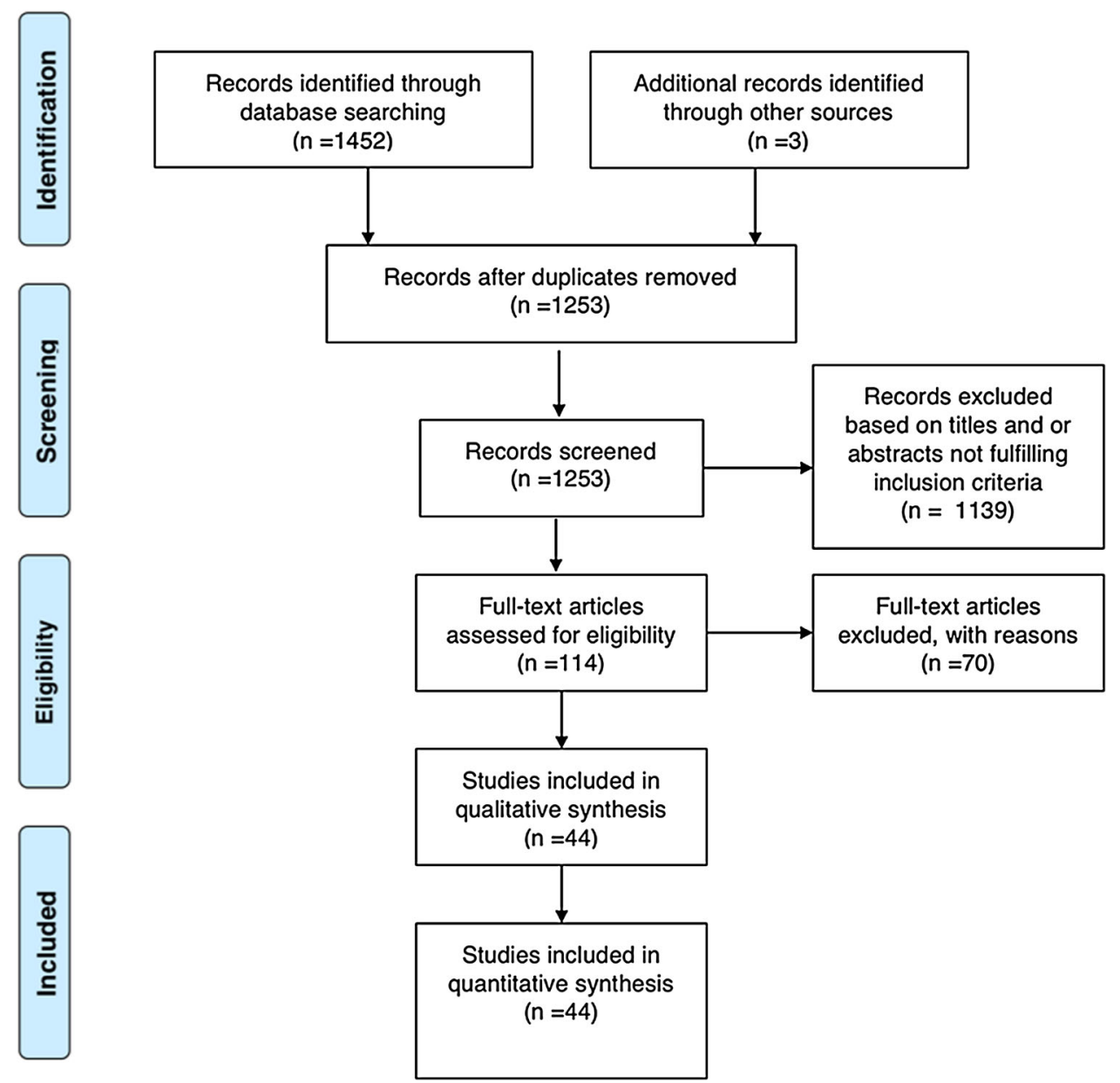

surgeons today to understand ideals of beauty and reproduce aesthetically 'beautiful' proportions for patients. To date, there is no agreed standard to measure facial beauty, and this remains a challenging task. It is a vital consideration for the aesthetic surgeon because there is a positive association between the outcomes of aesthetic surgery and better mental and psychological health, and therefore measuring outcomes quantitatively would facilitate this endeavour [42]. The aim of this investigation was to undertake a review to answer the question.

In the treatment of adults requesting facial aesthetic improvement, is there an evidence-based approach in quantitatively assessing beauty that is useful in everyday aesthetic practice?

Upon reviewing the data, it became evident that the diverse measurement criteria, methodologies used and population types in trials made it difficult to compare data. For example, different measurements from different types of photographic techniques would introduce photographic bias [43]. The trials have at best been of Level III or less for evidence, mainly being cross-sectional studies or observational studies. Despite these difficulties, some common themes were discovered and are highlighted below. These were related to lip analyses, eye measurements, symmetry, ethnicity, automation of analysis and the golden ratio.

\section{Lip Measurements}

Lip augmentation is one of the most common aesthetic procedures undertaken to correct age-related changes [44]. In the perception of beauty and attractiveness, measurements for individual facial features have been used. Lip measurements, for example, are known to influence an aesthetically youthful appearance [45]. Some authors define an 'ideal lip' as having good definition of the vermillion border with lower and upper lip balance [46]

Bagheri et al. undertook lip measurements in a Turkish population of 200 persons who were classified into of full, medium, thin and very thin type lips. They concluded there were significant gender differences in lip sizes and the aesthetic ranking of lips. Medium and full lip types were the significant proportion in males, and in females, medium was predominant. They also concluded that very thin lip types are rare in both sexes [62]. Heidekrueger et al. 


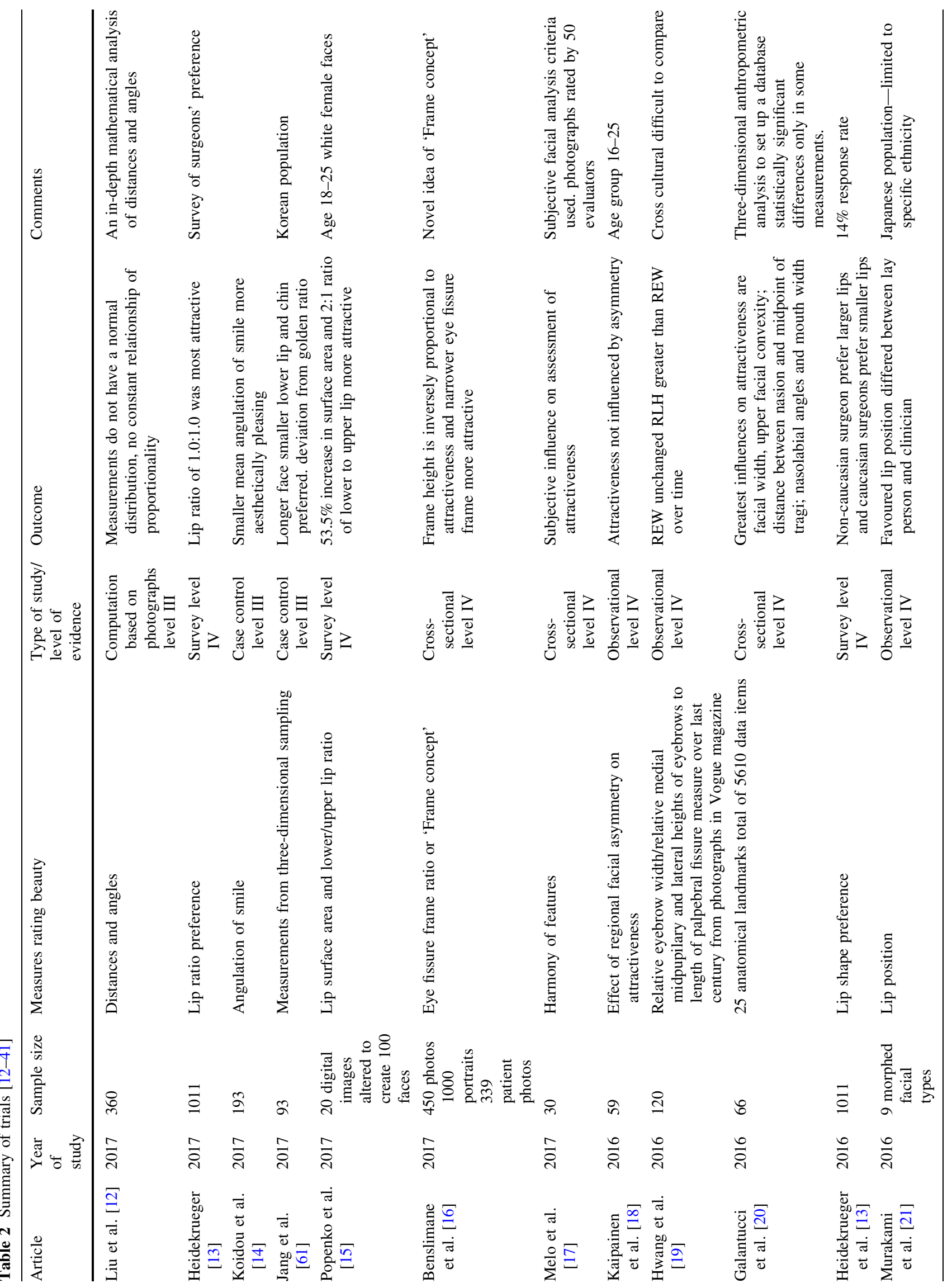




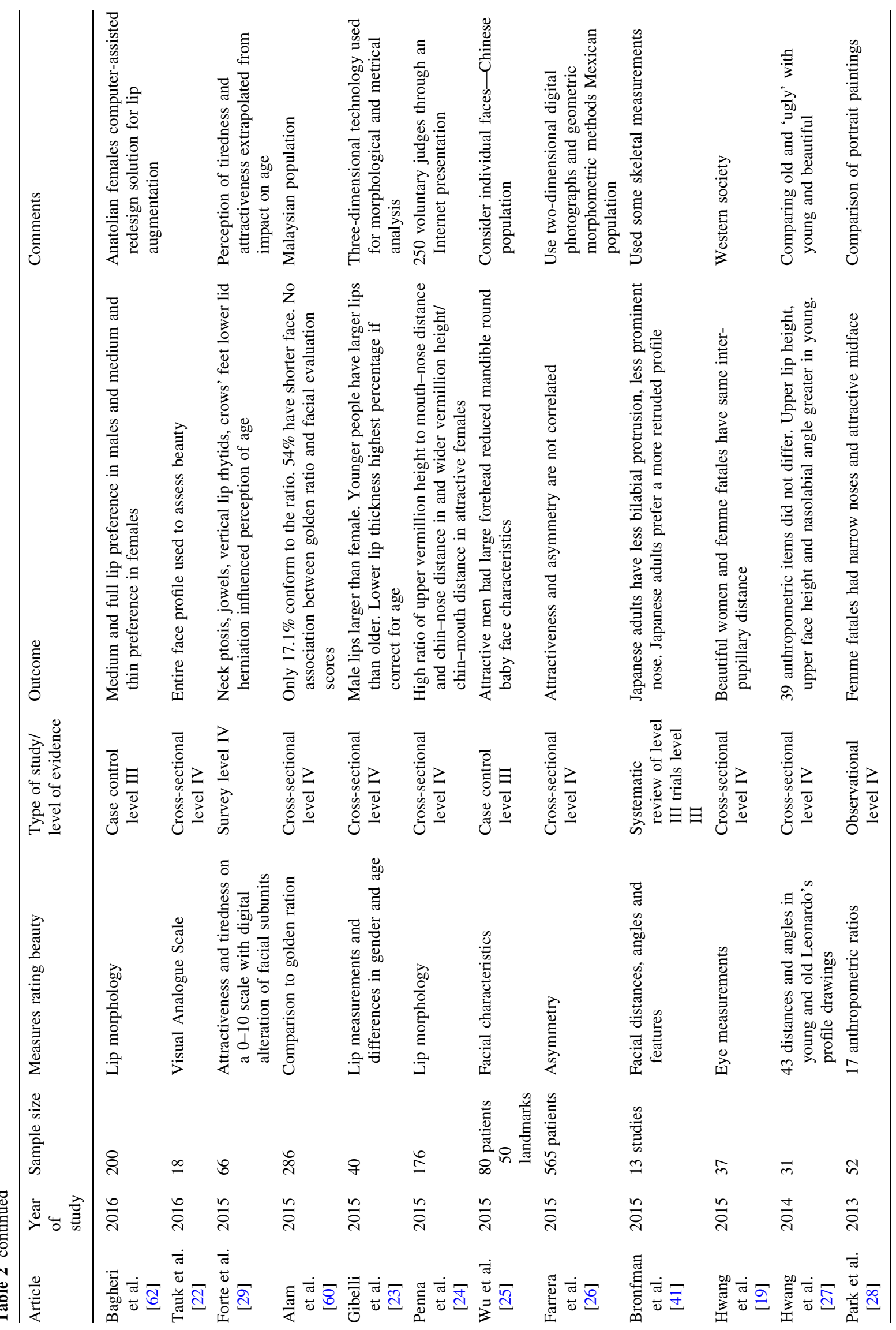




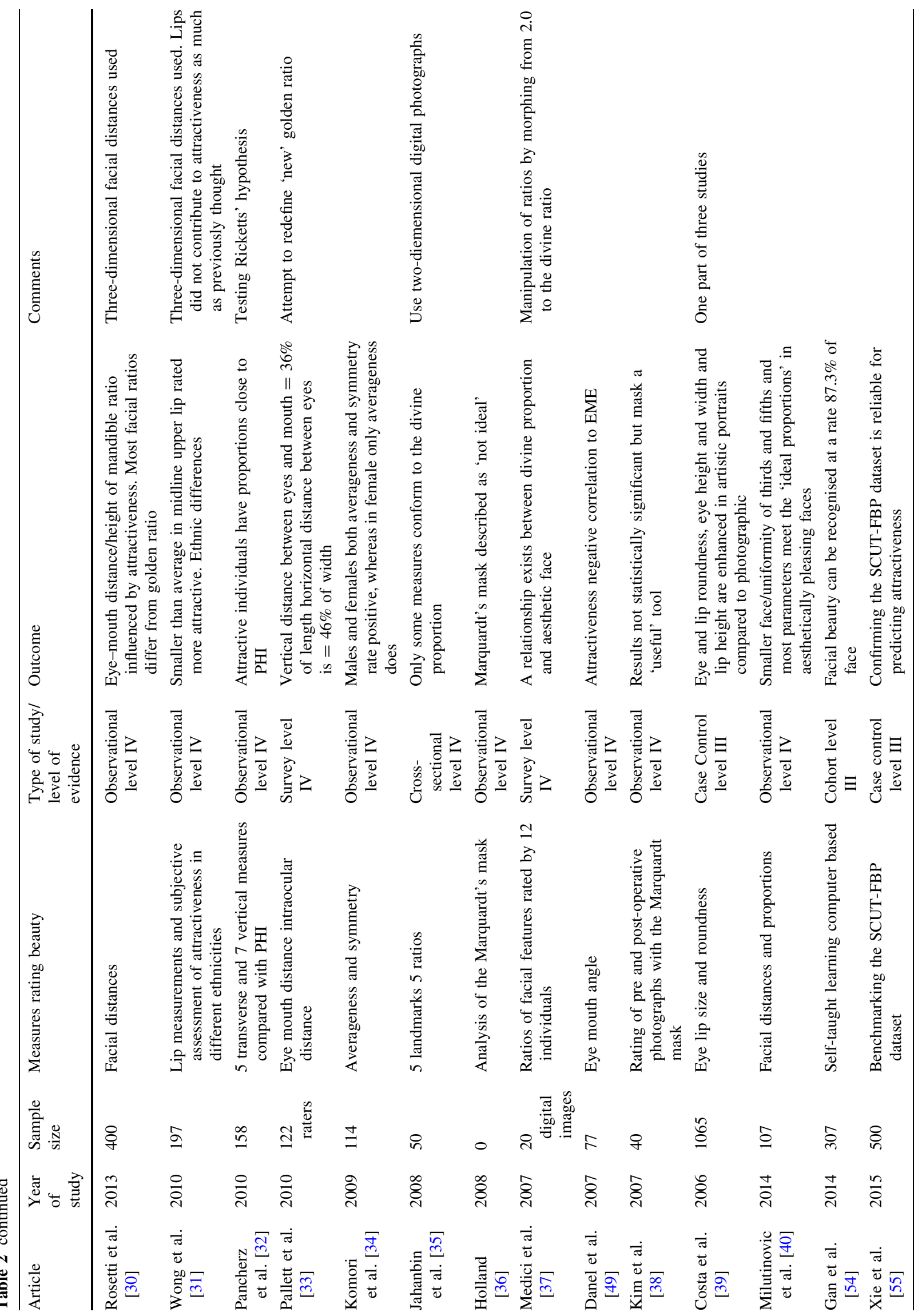


undertook a cross-sectional analysis of lip size preference through an online survey of 9000 plastic surgeons and lay persons. With a response rate of $14 \%$ from 35 different countries, they suggested the ethnicity, country of residence and profession had an impact on lip shape preference. They found that surgeons, who are non-Caucasian or who practise in Asia, have a preference for larger lips, whereas European and Caucasians prefer smaller lips. In the follow-up of this paper using the same responses, the team was able to assess the most popular lip ratio in the survey takers. A ratio of 1:1 was preferred in $60 \%$ of responders, whereas Popenko et al., assessing the attractiveness ranking of lip dimensions in 100 morphed faces of Caucasian women, suggested $53.5 \%$ increase in surface area from baseline and 2:1 ratio of lower to upper lip was the more attractive. Penna et al. found there was a higher ratio of upper vermillion height to mouth-nose distance and of chin-nose distance in attractive females.

These studies are not directly comparable due to the diverse measurements and populations but give us an insight into some lip preferences of patients and surgeons.

\section{Eyes}

Eye size, position, eyelid ptosis and eye 'frame' have all been related to perceptions of beauty.

Bensilmane introduced 'the Frame concept' to quantify and assess the characteristics of the female periorbital region. The author highlighted the fact that aesthetic practitioners most often analyse both upper and lower lids separately and rarely the gaze itself. The author strives to validate this ratio using anthropometric measures, to prove his hypothesis that the narrower the frame the more aesthetically pleasing [16]. As Benslimane et al. validate 'the frame' concept, the authors agree that a jaguar-like upward slant of the lower eyelid is more pleasing [47]. Photographs of models were analysed and frame anthropometry measured. The frame height was found to be inversely correlated with attractiveness, and this was synonymous for classical portraits of beautiful females.

Costa et al. reviewed photographs and historic artistic portraits and established that eye roundness, height and length were enhanced in artistic portraits, suggesting these features may be more beautiful. Larger eye size in proportion to the face has been shown to be more attractive in females [48]. Danel et al. identified that the eye-moutheye (EME) angle can be used as a quantitative measure of masculinity and fascial symmetry in males, which is independent of facial size. They concluded that there is a negative relationship between the EME angle and attractiveness. Hence, eye size, eyelid ptosis and frame contribute to beauty with a negative correlation with EME angle [49].

\section{Symmetry}

Kaipainen et al. assessed regional facial asymmetry and its influence on attractiveness. In their small sample size, most had some facial asymmetry, particularly in the lower and middle third of the face. The team did not find any association between regional asymmetry and attractiveness. Komori et al. suggested that the female facial symmetry does not appear to affect attractiveness. Their sample size was small, and therefore their conclusions may not be extrapolated. Farrera et al. undertook measurements from photographs of 565 Mexican individuals and chose a sample of 100, to rate for attractiveness after grouping into asymmetry variation. Their conclusion was also that symmetry does not affect attractiveness.

Other observers suggested that symmetry is important to facial beauty [50, 51]. Honn et al., for instance, argued that the symmetry has an influence on attractiveness [52].

Scientists and philosophers have traditionally attempted to appreciate attractiveness and beauty in terms of symmetry, and therefore, it may be that symmetry is not as important as previously thought, and perhaps beauty is related to proportions or ratios of the facial aesthetic units rather than to symmetry [53].

\section{Ethnicity}

We live in a heterogeneous society with persons from diverse backgrounds seeking aesthetic treatments. It is therefore important that the clinician is aware of average facial characteristics of different ethnic groups.

Bronfman's systematic review looked at 13 different studies focusing on the Japanese preference for aesthetic profile and concluded that Japanese males had smaller noses and bilabial protrusion, whereas females had more bilabial protrusion and a less prominent chin when compared with white populations. American and Japanese examiners favoured a lip profile that was retruded compared to African examiners. This suggests that consideration should be given to the ethnicity of the patient and that the clinician should be aware that one's own ethnic background may have an influence on the shared decision making during a consultation for aesthetic treatment.

\section{Technology and/or Automation}

Geometric evaluation of features and proportions is cumbersome and requires considerable investment of time. If the measurements can be predicted or calculated by software, a more rapid appraisal of beauty in the clinic setting is possible.

Gan et al. introduced a novel method for extracting facial features from images using an algorithm through 
machine learning [54]. This approach may avoid the likelihood of manual intervention. Although the idea of automated beauty recognition is novel, his paper utilizes simple non-detailed information such as curves and edges and is not concerned with the individual facial structures such as the eyebrows and nose. This prediction, however, is based on two-dimensional photographs, possibly lending itself to measurement inaccuracies and photograph bias. Xie et al. argued the case for a dataset of geometric measurements for application in facial beauty analysis [55] They gave attractiveness ratings using classical and deep learning methods to develop an algorithm, to learn and to predict facial beauty automatically. Galantucci et al., on the other hand, set out to verify a facial beauty prediction modelling method of principal component analysis (PCA) for measuring facial features for beauty classification [56]. The team used three-dimensional digital photogrammetry on real Miss Italy 2010 beauty contestants to confirm beauty ranking and PCA analysis to conclude that it is not a valid prediction tool. Mojallal et al.'s commentary paper appreciates the value of quantitative measurement of volume loss through the use of a three-dimensional camera. The team argued that the classical anthropometry measurements of the face are highly inaccurate and the 'differences in volume, distance, and projections' are too small to measure by these methods. They suggest objective evaluation, through the use of digital three-dimensional stereophotogrammetry, after facial rejuvenation allowing $360^{\circ}$ views of the individual. The advantage of this technique is that it allows for volume measurement as well as proportion measurement. The disadvantages are that the images require special manipulation and the technique is time consuming, and therefore it may not actually be useful in everyday practice [57]. Rossetti et al. also employed the use of three-dimensional stereophotogrammetry to investigate whether the 'golden' relationship exists between measurements of facial features [58]. They undertook measurements using reproducible three-dimensional techniques already described and validated [59]. Multiple measurements were undertaken, and the authors used previously acknowledged 'traditional' landmarks. Through their statistical analysis of the measurements, the team used ten ratios to compare with the golden ratio. Their analysis concluded there was no similarity to the golden ratio in their measurements.

Their study is important because they utilise three-dimensional technology for measurements, allowing easy calculation of the distance between facial landmarks. This gives a highly representative sample to work with. Threedimensional stereophotogrammetry in their study was useful to undermine the theory of the golden proportion, or golden ratio, in most measurements of facial proportion though it cannot be extrapolated to other ethnic groups.

\section{The Golden Ratio}

The concept of the golden ratio has been used since the time of Phidias, and its relation to aesthetic beauty still continues to be debated.

Alam et al. investigated the association of facial measurements with the golden ratio in a Malaysian population [60]. Using direct facial measurements from surface landmarks rather than from photographs is possibly more accurate. This cross-sectional study of 286 patients found that, in this population, only $17.1 \%$ of facial proportions correlated with the golden ratio and concluded that an association does not exist between the facial measurements in their Malaysian population and the golden ratio. Jahanbin et al. used 50 standardised profile silhouette photographs and 20 judges scored these on a VAS score. Measurements were made to assess whether any facial proportions fit the golden ratio. After assessing five landmarks and five ratios, none had the golden ratio mean of 1.618. Park et al., testing 17 anthropometric ratios for portraits of femme fatales, showed a midface ratio of $36 \%$ of the total face height. The proportions from portraits are closer to 'ideal' than in ratios measured clinically. Jang et al. undertook measurements from three-dimensional sampling of 93 patients in a Korean population and concluded that a longer face, smaller lip and chin size were preferable in females of a Korean population and this is a deviation from the golden ratio [61]. Milutinovic et al. assess different facial proportions and their relationship to attractiveness in Caucasians and any deviations from the ideal proportions or the divine ratio. In their group, they found that in attractive females, the divine ratio was met in three out of the six measured parameters. They establish that attractive females have facial proportions nearer to the divine ratio.

Medici et al. examined four ratios of frontal photographs of 20 Caucasian patients and concluded there is a relationship between the divine proportion close to or at a ratio of 1:1.618 and facial aesthetics. Kim et al. assessed the usefulness of the golden ratio and application through the use of the Marquardt's mask in forty cases of pre- and postoperative photographs. Scores were compared for the applied mask and for those without applied photographs and concluded that the Marquardt's mask was useful as an analytical tool for facial analysis, whereas Holland argued in his article that the Marquardt's mask is less than ideal. He states the methodology used to assess the fit of the mask for faces is 'faulty', that the mask approximates to a masculinised European female face and that it does not appear to approximate to a desired 'ideal' face. Undoubtedly, all this evidence suggests that the jury is still out on the usefulness of the golden ratio and that a consensus does not yet exist on this issue. 


\section{Conclusions}

There is an overwhelming desire to quantify beauty when planning aesthetic procedures in the light of increasing demand, and therefore an evidence-based approach is desirable. From the Greek scholars, through to the Renaissance polymaths, to today's three-dimensional predictive modelling, we have attempted to define and measure beauty. Despite measures of individual components such as fuller lip size and defined vermillion border, larger eyes and the 'Frame concept of eyes', through to the sum of all the parts, symmetry and the Golden Ratio, we are yet far from establishing what truly constitutes quantitative beauty.

It may ultimately be the case that measuring beauty may not provide great practical value because comparative measurements ignore the individuality in all of us. Perhaps as the famous poet Margaret Hungerford states that beauty is truly in the 'eyes of the beholder'.

\section{Compliance with Ethical Standards}

Conflict of interests The authors declare that they have no conflict of interest.

Open Access This article is distributed under the terms of the Creative Commons Attribution 4.0 International License (http:// creativecommons.org/licenses/by/4.0/), which permits unrestricted use, distribution, and reproduction in any medium, provided you give appropriate credit to the original author(s) and the source, provide a link to the Creative Commons license, and indicate if changes were made.

\section{References}

1. Marten T, Elyassnia D (2015) Fat grafting in facial rejuvenation. Clin Plast Surg 42(2):219-252

2. American Society of Plastic Surgeons (2017) National Clearinghouse of plastic surgery statistics. 2010 report of the 2009 statistics. Accessed 10th July 2017

3. American Society of Plastic Surgeons (2017) National Clearinghouse of plastic surgery statistics. 2016 report of the 2016 statistics. Accessed 1st July 2017

4. Faure JC, Rieffe C, Maltha JC (2002) The influence of different facial components on facial aesthetics. Eur J Orthod 24:1-7

5. Baldwin DC (1980) Appearance and aesthetics in oral health. Community Dent Oral Epidemiol 8:244-256

6. Barker DJ, Barker MJ (2002) The body as art. J Cosmet Dermatol $1: 88-93$

7. Bashour M (2006) History and current concepts in the analysis of facial attractiveness. Plast Reconstr Surg 118:741-756

8. Adamson PA, Zavod MB (2006) Changing perceptions of beauty: a surgeon's perspective. Fac Plast Surg 22:188-193

9. Larrabee WF (1997) Facial beauty Myth or reality? Arch Otolaryngol Head Neck Surg 123(6):571-572

10. Foo P, Sampson W, Roberts R, Jamieson L, David D (2013) Facial aesthetics and perceived need for further treatment among adults with repaired cleft as assessed by cleft team professionals and laypersons. The Eur J Orthod 35(3):341-346
11. Huang X, Lin J, Demner-Fushman D (2006) Evaluation of PICO as a knowledge representation for clinical questions. AMIA Ann Symp Proc 2006:359-363

12. Liu S, Fan YY, Guo Z, Samal A, Ali A (2017) A landmark-based data-driven approach on 2.5D facial attractiveness computation. Neurocomputing 238:168-178

13. Heidekrueger P, Juran S, Szpalski C, Larcher L, Ng R et al (2017) The current preferred female lip ratio. J Cranio-Maxillofac Surg 45(5):655-660

14. Koidou VP, Rosenstiel SF, Rashid RG (2017) Celebrity smile esthetics assessment: smile angulation. J Prosthet Dent 117(5):636-641. https://doi.org/10.1016/j.prosdent.2016.08.008

15. Popenko NA, Tripathi PB, Devcic Z, Karimi K, Osann K, Wong BJF (2017) A quantitative approach to determining the ideal female lip aesthetic and its effect on facial attractiveness. JAMA Fac Plast Surg 19(4):261-267. https://doi.org/10.1001/jamafacial. 2016.2049

16. Benslimane F, van Harpen L, Myers SR, Ingallina F, Ghanem AM (2017) The Benslimane's artistic model for females' gaze beauty: an original assessment tool. Aesthetic Plast Surg 41(1):81-89

17. Melo AR, Conti ACCF, Almeida-Pedrin RR, Didier V, Valarelli DP, Capelozza Filho L (2017) Evaluation of facial attractiveness in black people according to the subjective facial analysis criteria. Dent Press J Orthod 22(1):75-81. https://doi.org/10.1590/21776709.22.1.075-081.oar

18. Kaipainen A, Sieber R, Nada R, Maal T, Katsaros C, Fudalej P (2016) Regional facial asymmetries and attractiveness of the face. Eur J Orthod 38(6):602-608

19. Hwang K, Hong JU, Park SJ, Hwang SW (2015) Eyes of beautiful women and femme fatales depicted in western portraits. J Cranio Surg 26(3):937-939

20. Galantucci LM, Di Gioia E, Lavecchia F, Percoco G (2014) Is principal component analysis an effective tool to predict face attractiveness? A contribution based on real 3D faces of highly selected attractive women, scanned with stereophotogrammetry. Med Biol Eng Comput 52(5):475-489. https://doi.org/10.1007/ s11517-014-1148-8

21. Murakami T, Kataoka T, Tagawa J, Yamashiro T, Kamioka H (2016) Antero-posterior and vertical facial type variations influence the aesthetic preference of the antero-posterior lip positions. The Eur J Orthod 38(4):414-421. https://doi.org/10.1093/ejo/ cjv073

22. Tauk A, Bassil-Nassif N, Mouhanna-Fattal C, Bouserhal J (2016) The importance of using the entire face to assess facial profile attractiveness. Int Orthod 14(1):65-79. https://doi.org/10.1016/j. ortho.2015.12.007

23. Gibelli D, Codari M, Rosati R, Dolci C, Tartaglia GM, Cattaneo C, Sforza C (2015) A quantitative analysis of lip aesthetics: the influence of gender and aging, Aesthetic Plast Surg 39(5):771-776. doi: 10.1007/s00266-015-0495-7. Epub 2015 May 7. Erratum in: Aesthetic Plast Surg. 2015 Oct; 39(5):777

24. Penna V, Fricke A, Iblher N, Eisenhardt SU, Stark GB (2015) The attractive lip: a photomorphometric analysis. J Plast Reconstr Aesthet Surg 68(7):920-929

25. Wu F, Li J, He H, Huang N, Tang Y, Wang Y (2015) Soft-tissue facial characteristics of attractive Chinese men compared to normal men. Int J Clin Exp Med 8(5):7977-7982

26. Farrera A, Villanueva M, Quinto-Sánchez M, González-José R (2015) The relationship between facial shape asymmetry and attractiveness in Mexican students. Am $J$ Hum Biol 27(3):387-396. https://doi.org/10.1002/ajhb.22657

27. Hwang K, Heo WY, Jeong JM, Hwang SW (2014) Anthropometric comparison of the idealized youth and hideous old man of Leonardo's profile drawings. J Craniofac Surg 25(6):2223-2226 
28. Park HS, Rhee SC, Kang SR, Lee JH (2004) Harmonized profiloplasty using balanced angular profile analysis. Aesthet Plast Surg 28(2):89-97. https://doi.org/10.1007/s00266-004-3125-3

29. Fortes HNDR, Guimarães TC, Belo IML, Matta ENRD (2014) Photometric analysis of esthetically pleasant and unpleasant facial profile. Dent Press J Orthod 19(2):66-75

30. Rossetti A, De Menezes M, Rosati R, Ferrario VF, Sforza C (2013) The role of the golden proportion in the evaluation of facial esthetics. Angle Orthod 83(5):801-808

31. Wong BJF, Karimi K, Devcic Z, McLaren CE, Chen W-P (2008) Evolving attractive faces using morphing technology and a genetic algorithm: a new approach to determining ideal facial aesthetics. The Laryngoscope 118(6):962-974. https://doi.org/10. 1097/MLG.0b013e31816bf545

32. Pancherz H (2010) Divine proportions in attractive and nonattractive faces. World J Orthod 11(1):27

33. Pallett PM, Link S, Lee K (2010) New "golden" ratios for facial beauty. Vis Res 50(2):149-154

34. Komori M, Kawamura S, Ishihara S (2009) Averageness or symmetry: Which is more important for facial attractiveness? Acta Psychol 131(2):136-142. https://doi.org/10.1016/j.actpsy. 2009.03.008

35. Jahanbin A (2008) The effects of upper lip height on smile esthetics perception in normal occlusion and nonextraction, orthodontically treated females. Indian J Dent Res 19:204

36. Holland E (2008) Marquardt's phi mask: pitfalls of relying on fashion models and the golden ratio to describe a beautiful face. Aesthet Plast Surg 32(2):200-208

37. Medici Filho E, Martins MV, dos Santos da Silva MA, de M Castilho JC, de Moraes LC, de A Gil CTL (2007) Divine proportions and facial esthetics after manipulation of frontal photographs. World J Orthod 8(2):103-108

38. Kim Y-H (2007) Easy facial analysis using the facial golden mask. The J Craniofac Surg 18(3):643-649. https://doi.org/10. 1097/scs.0b013e3180305304

39. Costa M, Corazza L (2006) Aesthetic phenomena as supernormal stimuli: the case of eye, lip, and lower-face size and roundness in artistic portraits. Perception 35(2):229-246

40. Milutinovic J, Zelic K, Nedeljkovic N (2014) Evaluation of facial beauty using anthropometric proportions. The Sci World J 2014:1-8. https://doi.org/10.1155/2014/428250

41. Bronfman CN, Janson G, Pinzan A, Rocha TL (2015) Cephalometric norms and esthetic profile preference for the Japanese: a systematic review. Dent Press J Orthod 20(6):43-51

42. Gillen MM (2015) Associations between positive body image and indicators of men's and women's mental and physi- cal health. Body Image 13:67-74

43. Strub B, Mende K, Meuli-Simmen C, Bessler S (2015) The frontal view of the nose: lighting effects and photographic bias. Aesthet Surg J 35(5):524-532

44. Smith S, Lin X, Shamban A (2013) Small gel particle hyaluronic acid injection technique for lip augmentation. J Drugs Dermatol 12(7):764-769

45. AlamM Gladstone H, Kramer E et al (2008) ASDS guidelines of care: injectable fillers. Dermatol Surg 34(s1):S115-S1485
46. Garcia de Mitchell C, Pessa J, Schaverien M, Rohrich R (2008) The Philtrum: anatomical observations from a new perspective. Plast Reconstr Surg 122(6):1756-1760

47. Grundl M, Klein S, Horczakiwskyj R et al (2008) The jaguar's eye as a new beauty trend? Age related effects in judging the attractiveness of the oblique eye axis. Aesthet Plast Surg 32:915-919

48. Johnston VS, Franklin M (1993) Is beauty in the eye of the beholder? Ethol Sociobiol 14(3):183-199. https://doi.org/10. 1016/0162-3095(93)90005-3

49. Danel D, Pawlowski B (2007) Eye-mouth-eye angle as a good indicator of face masculinization, asymmetry, and attractiveness (Homo sapiens). J Comp Psychol 121(2):221-225

50. Rhodes G, Sumich A, Byatt G (1999) Are average facial configurations attractive only because of their symmetry? Psychol Sci 10(1):52-58. https://doi.org/10.1111/1467-9280.00106

51. Mealey L, Bridgstock R, Townsend GC (1999) Symmetry and perceived facial attractiveness: a monozygotic co-twin comparison. J Personal Soc Psychol, 76(1):151-8. Retrieved from http:// www.ncbi.nlm.nih.gov/pubmed/9972560

52. Hönn M, Göz G (2007) The ideal of facial beauty: a review. J Orofac Orthop Fortschr Der Kieferorthopädie 68(1):6-16. https://doi.org/10.1007/s00056-007-0604-6

53. Prokopakis EP, Vlastos IM, Picavet VA, Nolst Trenite G, Thomas R, Cingi C, Hellings PW (2013) The golden ratio in facial symmetry. Rhinology 51(1):18-21. https://doi.org/10.4193/ Rhino12.111

54. Gan J, Li L, Zhai Y, Liu Y (2014) Deep self-taught learning for facial beauty prediction. Neurocomputing 144:295-303. https:// doi.org/10.1016/j.neucom.2014.05.028

55. Xie D, Liang L, Jin L, Xu J, Li M (2015) SCUT-FBP: a benchmark dataset for facial beauty perception. In: 2015 IEEE international conference on systems, man, and cybernetics IEEE, pp. 1821-1826. http://doi.org/10.1109/SMC.2015.319

56. Shlens J (2014) A tutorial on principal component analysis. Retrieved from http://arxiv.org/abs/1404.1100

57. Ras F, Habets LL, van Ginkel FC, Prahl-Andersen B (1996) Quantification of facial morphology using stereophoto- grammetry-demonstration of a new concept. J Dent 24(5):369-374

58. Rossetti A, De Menezes M, Rosati R, Ferrario VF, Sforza C (2013) The role of the golden proportion in the evaluation of facial esthetics. The Angle Orthod 83(5):801-808

59. Rosati R, Rossetti A, De Menezes M, Ferrario VF, Sforza C (2012) The occlusal plane in the facial context: inter-operator repeatability of a new three-dimensional method. Int J Oral Sci 4:34-37

60. Alam MK, Mohd Noor NF, Basri R, Yew TF, Wen TH (2015) Multiracial facial golden ratio and evaluation of facial appearance. PLoS ONE 10(11):e0142914

61. Jang KS, Bayome M, Park JH, Park K-H, Moon H-B, Kook Y-A (2017) A three-dimensional photogrammetric analysis of the facial esthetics of the Miss Korea pageant contestants. Korean J Orthod 47(2):87-99

62. Bagheri H, Sirinturk S, Govsa F, Pinar Y, Ozer MA (2017) Digitalized analysis of philtral anatomy for planning individual treatment. Surg Radiol Anat 39(11):1183-1189 